OPEN ACCESS

Edited by:

Jens Staal,

Ghent University, Belgium

Reviewed by:

José Díaz,

University of A Coruña, Spain

Fei Gao,

University of Arkansas, United States

*Correspondence:

Yuwen Yang

ywyang92@hotmail.com

Specialty section

This article was submitted to

Plant Microbe Interactions,

a section of the journal

Frontiers in Plant Science

Received: 06 July 2017 Accepted: 29 December 2017 Published: 17 January 2018

Citation:

Yang $Y$, Chen $T$, Ling $X$ and $M a Z$ (2018) Gbvdr6, a Gene Encoding a Receptor-Like Protein of Cotton

(Gossypium barbadense), Confers Resistance to Verticillium Wilt in Arabidopsis and Upland Cotton.

Front. Plant Sci. 8:2272. doi: 10.3389/fp/s.2017.02272

\section{Gbvdr6, a Gene Encoding a Receptor-Like Protein of Cotton (Gossypium barbadense), Confers Resistance to Verticillium Wilt in Arabidopsis and Upland Cotton}

\author{
Yuwen Yang ${ }^{1,2 \star}$, Tianzi Chen ${ }^{2}$, Xitie Ling $^{2}$ and Zhengqiang $\mathrm{Ma}^{1}$ \\ ${ }^{1}$ The Applied Plant Genomics Laboratory of Crop Genomics and Bioinformatics Center, National Key Laboratory of Crop \\ Genetics and Germplasm Enhancement, Nanjing Agricultural University, Nanjing, China, ${ }^{2}$ Provincial Key Laboratory of \\ Agrobiology, Jiangsu Academy of Agricultural Sciences, Nanjing, China
}

Verticillium wilt is a soil-borne disease that can cause devastating losses in cotton production. Because there is no effective chemical means to combat the disease, the only effective way to control Verticillium wilt is through genetic improvement. Therefore, the identification of additional disease-resistance genes will benefit efforts toward the genetic improvement of cotton resistance to Verticillium wilt. Based on screening of a BAC library with a partial Ve homologous fragment and expression analysis, a $V$. dahliae-induced gene, Gbvdr6, was isolated and cloned from the Verticillium wilt-resistant cotton G. barbadense cultivar Hai7124. The gene was located in the gene cluster containing Gbve1 and Gbvdr5 and adjacent to the Verticillium wilt-resistance QTL hotspot. Gbvdr6 was induced by Verticillium dahliae Kleb and by the plant hormones salicylic acid (SA), methyl jasmonate (MeJA) and ethephon (ETH) but not by abscisic acid (ABA). Gbvdr6 was localized to the plasma membrane. Overexpression of Gbvdr6 in Arabidopsis and cotton enhanced resistance to $\mathrm{V}$. dahliae. Moreover, the JA/ET signaling pathway-related genes PR3, PDF 1.2, ERF1 and the SA-related genes PR1 and PR2 were constitutively expressed in transgenic plants. Gbvdr6-overexpressing Arabidopsis was less sensitive than the wild-type plant to MeJA. Furthermore, the accumulation of reactive oxygen species and callose was triggered at early time points after $V$. dahliae infection. These results suggest that Gbvdr6 confers resistance to $V$. dahliae through regulation of the JA/ET and SA signaling pathways.

Keywords: Gbvdr6, Gossypium, resistance, signaling pathway, Verticillium wilt

\section{INTRODUCTION}

Gossypium hirsutum (also known as upland or Mexican cotton) is the most widely cultivated cotton species in the world and is generally susceptible to Verticillium wilt, which is one of the most destructive diseases caused by the soil-borne fungus Verticillium dahliae Kleb (Zhou et al., 2013; Zhang J. et al., 2014). In contrast, Gossypium barbadense (also known as Sea Island, Pima, or Egyptian cotton) is usually resistant or tolerant to Verticillium wilt (Zhang J. et al., 2014). The genetic improvement of $G$. hirsutum through introgression from G. barbadense is an effective way 
to control Verticillium wilt (Zhang J. et al., 2012; Fang et al., 2013; Zhou et al., 2013). However, hybrid breakdown and sterility in interspecific crosses between G. hirsutum and G. barbadense are real obstacles to the efficient use of Verticillium wilt resistance from G. barbadense (Zhang J. et al., 2014). In addition to conventional breeding, the genetic engineering of diseaseresistance genes is an effective means of controlling Verticillium wilt in cotton (Wang et al., 2004; Rajasekaran et al., 2005; Tohidfar et al., 2005; Miao et al., 2010; Parkhi et al., 2010; Tian et al., 2010). By screening a cotton bacterial artificial chromosome library using a cotton-expressed sequence tag that shares $48 \%$ similarity with the Verticillium wilt-resistance gene $\mathrm{Ve1}$, we identified forty independent positive clones and isolated two Verticillium wilt-resistance genes encoding receptor-like proteins (RLPs) (Zhang B. et al., 2012; Yang et al., 2015a). Other proteins, including mitogen-activated protein kinases, WRKY transcription factors, MYB transcription factors, major latex proteins, polyamine oxidases, receptor-like kinase, BRI1associated receptor kinase, and subtilase are also involved in the defense response of cotton to V. dahliae (Gao et al., 2013; Li et al., 2014; Zhang X. et al., 2014; Jun et al., 2015; Mo et al., 2015; Yang et al., 2015a; Cheng et al., 2016; Duan et al., 2016). However, the control of Verticillium wilt is extremely difficult due to the persistence of the microsclerotia in soil and the lack of an effective chemical means of combating the pathogens (Klosterman et al., 2009). Therefore, the identification of additional disease-resistance genes will benefit efforts in the genetic improvement of cotton's resistance to Verticillium wilt.

RLPs, two examples of which are the tomato Cladosporium fulvum ( $C f$ ) resistance genes and the tomato Verticillium wiltresistance Ve locus (Kawchuk et al., 2001; Kruijt et al., 2005), typically possess an extracellular leucine-rich repeat (eLRR), a single transmembrane domain, and a short cytoplasmic C terminus. The Ve locus comprises two closely linked inversely oriented genes, $\mathrm{Ve} 1$ and $\mathrm{Ve} 2$, that share $84 \%$ amino acid identity. Whereas Ve1 mediates Verticillium resistance by recognizing the $V$. dahliae Ave1 effector, $\mathrm{Ve} 2$ has no resistance function in tomato or transgenic Arabidopsis (Fradin et al., 2009, 2011; Jonge et al., 2012). Domain swaps between Ve1 and Ve2 showed that the first 30 eLRRs of Ve1 can be replaced by those of Ve2, whereas the eLRR30-eLRR35 region and the C-terminus of Ve1 are crucial for Verticillium wilt resistance and cannot be replaced by those of Ve2 (Fradin et al., 2014). Mutational analysis of Ve1 further revealed that the C1 domain eLRR1-eLRR8 and eLRR20eLRR23, the C2 domain, and the C3 domain eLRR32-eLRR37 are required for Ve1 functionality, whereas the GxxxG motif in the transmembrane domain and two putative endocytosis motifs in the C-terminus are not (Zhang Z. et al., 2014). The chaperones HSP70 binding proteins (BiPs) and a lectin-type calreticulin (CRT) were verified to be involved in Ve1-mediated resistance to Verticillium (Liebrand et al., 2014).

In response to infection by pathogens, plants have evolved a series of inducible defenses, including the induction of the hypersensitive response (HR), the formation of reactive oxygen species (ROS), the deposition of callose, and the production and accumulation of antimicrobial proteins, phytoalexins, and PR proteins (Luo et al., 2014). Ve1-mediated Verticillium wilt resistance triggers an $\mathrm{HR}$ in tomato (Solanum lycopersicum) and Nicotiana tabacum (Jonge et al., 2012; Zhang et al., 2013a), generates hydrogen peroxide $\left(\mathrm{H}_{2} \mathrm{O}_{2}\right)$ and increases the activities of peroxidase, phenylalanine ammonia lyase, and lignins (Gayoso et al., 2010). The overexpression of other RLPs and polyamine oxidase genes in transgenic Arabidopsis plants has also been shown to increase the levels of callose, $\mathrm{H}_{2} \mathrm{O}_{2}$, salicylic acid and phytoalexin during V. dahliae infection (Zhang B. et al., 2012; Mo et al., 2015; Yang et al., 2015a).

Genes with homology to $V e$ have been identified and cloned in our laboratory using screening of BAC clones combined with the genome walking method (Zhang B. et al., 2012; Yang et al., 2015b). A partial fragment of a $V e$-homologous gene was amplified from the genomic DNA of H7124 using primers designed according to a cotton EST (TC121084) in the gene index. The amplified sequence was subsequently used as a probe to screen a G. hirsutum cv. Maxxa BAC library, and 40 positive clones were identified (Tomkins et al., 2001). The expression patterns of these genes after inoculation of the plants with $V$. dahliae were analyzed by qRT-PCR, and Gbvdr6 was found to be activated. Therefore, Gbvdr6 was considered to confer resistance to $V$. dahliae and was chosen for further analysis. Gbvdr6-overexpressing Arabidopsis and cotton showed enhanced resistance to $V$. dahliae. Interestingly, the transgenic plants were less sensitive than wild-type plants to MeJA, while at the same time, the JA/ET signaling pathway was induced. $\mathrm{H}_{2} \mathrm{O}_{2}$ production and callose deposition were also found to be enhanced in Gbvdr6 transgenic plants at the early infection stage.

\section{MATERIALS AND METHODS}

\section{Plants, V. dahliae Strain and Inoculation Method}

Seedlings of the G. barbadense cultivar Hai7124, which is highly resistant to $V$. dahliae (Yang et al., 2008), were grown in chambers under greenhouse conditions. The Arabidopsis thaliana ecotype Columbia-0 was cultured in pots under controlled conditions (temperatures of $25^{\circ} \mathrm{C}$ during the day and $20^{\circ} \mathrm{C}$ at night, $60-70 \%$ relative humidity, and light intensity of $200 \mu \mathrm{mol} / \mathrm{m}^{-2} / \mathrm{s}^{-1}$ in a $16 / 8 \mathrm{~h}$ photoperiod). A non-defoliating isolate of $V$. dahliae, BP2 (Yang et al., 2008), was activated on potato dextrose agar and cultured in liquid Czapek medium at $25^{\circ} \mathrm{C}$. Before inoculation, the spore concentration of $V$. dahliae was determined by counting its spores under a microscope. For $V$. dahliae inoculation, seedlings of Hai7124 at the 2-leaf stage were inoculated by root irrigation with $10 \mathrm{~mL}$ of liquid containing $1 \times 10^{7}$ spores per pot (Zhang B. et al., 2012); plantlets of Arabidopsis thaliana were uprooted and dipped for $1 \mathrm{~min}$ in a suspension containing $1 \times 10^{7}$ spores $/ \mathrm{mL}$ and replanted in vermiculite (Fradin et al., 2011). For hormone treatment, the roots of cotton seedlings at the 4-leaf stage were immersed in $1 \mathrm{mM}$ salicylic acid (SA), $5 \mathrm{mM}$ ethephon (ETH), $100 \mu \mathrm{M}$ abscisic acid (ABA) or $100 \mu \mathrm{M}$ methyl jasmonate (MeJA), (Chen et al., 2014; Camacho-Cristóbal et al., 2015). Stem tissues from $V$. dahliae-infected plants and root tissues from hormone-treated plants were harvested at appropriate times for RNA extraction. 


\section{Isolation of Gbvdr6}

The gene cloning method used in this study followed our previously described protocol (Zhang B. et al., 2012; Yang et al., 2015a). Briefly, forty BAC clones were obtained by screening a $G$. hirsutum cv. Maxxa BAC library against a cotton EST (TC121084) that is highly homologous to the tomato Vel gene (Zhang B. et al., 2012). Partial sequences of the $V e 1$-homologous gene in one BAC clone were amplified using the conserved primers VeF1008 (5'-ttgagcaattgacaagaa tagagct- $\left.3^{\prime}\right)$ and VeR2731 (5'-tgaccctgtgaaagcattatgtga- $\left.3^{\prime}\right)$, and the full sequence of this BAC clone was obtained by genome walking using the specific primers $5^{\prime}$-ttagtgtaagtaagactgagggaag$3^{\prime}$ and $5^{\prime}$-gcaactcggtcagtggcaataaa- $3^{\prime}$ and $5^{\prime}$-ttcccaatgtccgtgtttga act- $3^{\prime}$ and $5^{\prime}$-ggcaaatcccaattcttaaccca- $3^{\prime}$ for $5^{\prime}$ and $3^{\prime}$ sequence amplification, respectively. To clone its ortholog in G. barbadense cv. Hai7124, the specific primers F-XbaI (5'-tgatctagactcaacct agtgccattgttatc- $\left.3^{\prime}\right)$ and R-SmaI (5'-tgacccgggaatcccaatagttgctagg

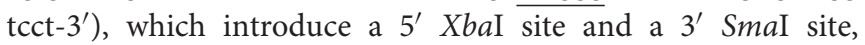
respectively, were designed according to the full sequences of the Ve1-homologous gene in G. hirsutum. Polymerase chain reaction (PCR) was conducted with Hai7124 cDNA and DNA as templates, respectively. The PCR program consisted of $3 \mathrm{~min}$ at $94^{\circ} \mathrm{C}$ followed by 32 cycles of denaturation for $45 \mathrm{~s}$ at $94^{\circ} \mathrm{C}$, annealing for $45 \mathrm{~s}$ at $56^{\circ} \mathrm{C}$, and extension for $3 \mathrm{~min}$ at $72^{\circ} \mathrm{C}$. The PCR product was cloned into the pGEM-T vector (Promega) and sequenced after agarose gel electrophoresis and purification using a QIAquick PCR Purification Kit (Qiagen).

Alignments of nucleotide and amino acid sequences were conducted using BioEdit software. A GenBank BLASTX search was performed on the website of the National Center for Biotechnology Information (http://www.ncbi.nlm.nih.gov). Phylogenetic analysis was performed using MEGA6 software (Tamura et al., 2013). The putative motif and domains were analyzed with SignalP 4.1 (http://www.cbs.dtu.dk/services/ SignalP/), InterProScan (http://www.ebi.ac.uk/interpro/ scan.html), and SMART (http://smart.embl-heidelberg.de/). The sequences of the SSR markers that flank Verticillium wilt-resistance quantitative trait loci (QTL) in cotton were downloaded from the CottonGen (https://www.cottongen.org) and CottonQTLdb (http://www2.cottonqtldb.org:8081/search) websites. The physical locations of genes and markers were determined by anchoring their sequences onto the island cotton upland cotton (G. hirsutum) cv. TM-1 genome (Zhang et al., 2015) through BLASTN with an $E$-value $=1.0 \mathrm{E}$-100 for genes and an $E$-value $=1.0$ for markers. To confirm the physical positions of the marker primers, the distance between one forward primer hit and one reverse primer hit was determined to be within 100-500 bp in the same chromosome.

\section{Expression Pattern Analysis of Gbvdr6}

To characterize the expression patterns of the Gbvdr6 gene in various tissues in response to $V$. dahliae infection and phytohormones, total RNA was extracted from root, stem and leaf tissues using an RNAiso Kit (TaKaRa) and transcribed into cDNA using a Primscript RT-PCR kit (TaKaRa) according to the manufacturer's instructions. A quantitative reverse transcription-polymerase chain reaction (qRT-PCR) was performed using a SYBR Premix ExTaqTM II Kit (TaKaRa) in a real-time PCR thermal cycler (qTOWER 2.0/2.2, Analytik Jena, Germany). The Gbvdr6-specific primers for qRT-PCR were F2952 (5'-tcgtcaccacctaaagaagacag- $\left.3^{\prime}\right)$ and R3097 (5'-cacgatcg acacgctcaaaatac- $\left.3^{\prime}\right)$, which corresponded to the $3^{\prime}$ end region of Gbvdr6 mRNA. The cotton polyubiquitin 14 gene served as an internal control, with the specific primers $5^{\prime}$-caacgctccatcttg tcctt- $3^{\prime}$ and $5^{\prime}$-tgatcgtctttccotaagc- $3^{\prime}$ (Artico et al., 2010). The PCR program consisted of an initial denaturation step of $1 \mathrm{~min}$ at $95^{\circ} \mathrm{C}$ followed by denaturation for $15 \mathrm{~s}$ at $95^{\circ} \mathrm{C}$, annealing for $20 \mathrm{~s}$ at $60^{\circ} \mathrm{C}$, and extension for $20 \mathrm{~s}$ at $72^{\circ} \mathrm{C}$ for 40 cycles. All $\mathrm{qRT}$-PCR results are expressed as the relative expression levels determined using three biological replicates. The Gbvdr6 promoter cassette was constructed by inserting the $1.66-\mathrm{Kb}$ fragment upstream of the start codon of Hai7124 into PbI101 using the primers pGbvdr6HinIIIF ( $5^{\prime}$-tccaagcttcagacttaccagg agataacattc- $\left.3^{\prime}\right)$ and pGbvdr6BamHIR (5'-agtggatccaatacaacaa agaattatgaagaa- $\left.3^{\prime}\right)$. Histochemical localization of GUS activity in Arabidopsis transfected with the Gbvdr6 promoter construct was performed as previously described (Jefferson et al., 1987). The relative expression levels were calculated using the $2^{-\Delta \Delta C T}$ method (Livak and Schmittgen, 2001). The relative transcript levels of Gbvdr6 were normalized to the transcript levels of the polyubiquitin 14 (UBQ14) gene. In each case, three technical replicates were performed for each of at least three independent biological replicates.

\section{Subcellular Localization Analysis of Gbvdr6}

Gbvdr6 was PCR-amplified using the primers $5^{\prime}$-cggggtaccatg aggatttcactcttttc- $3^{\prime}$ and $5^{\prime}$-cgcggatccggtcctcctttggttctg- $\overline{3^{\prime} \text {, which }}$ introduce a $5^{\prime} \mathrm{Kp} n \mathrm{I}$ site and a $3^{\prime} \mathrm{Ba} \mathrm{mHI}$ site, respectively, and fused to the N-terminus of GFP in the pBinGFP4 vector under the control of the CaMV35S promoter (Liu et al., 2014). The Gbvdr6-GFP fusion and the plasma membrane marker mCherry (Nelson et al., 2007) were co-agroinfiltrated into N. benthamiana leaves. Fluorescence was imaged at $48 \mathrm{~h}$ post-infiltration using a Zeiss LSM710 confocal microscope (Zeiss Microsystems) at specific excitation and emission wavelengths (GFP, 488 and 495$530 \mathrm{~nm}$; mCherry, 587 and 600-650 nm).

\section{Pathogen Inoculation Assay of Transgenic Arabidopsis and Cotton}

A binary vector containing an overexpression cassette of $G b v d r 6$ under control of the CaMV35S promoter was transformed into Arabidopsis Columbia and Gossypium hirsutum var. 03298 by floral dip and Agrobacterium-mediated transformation of cotton hypocotyl, respectively (Umbeck et al., 1987; Bent, 2006). The expression of Gbvdr6 in transgenic plants was investigated by qRT-PCR using the specific primers F1785 (5'-gcaacaagtctcga gtacctaaat- $\left.3^{\prime}\right)$ and R2140 (5'-ccaagagacacgttcatctgaaag- $\left.3^{\prime}\right)$. The Arabidopsis $\beta$-tubulin gene and the cotton polyubiquitin 14 gene were used as internal controls (Hiratsu et al., 2003).

The Verticillium wilt resistance of T3 transgenic Gbvdr6 Arabidopsis was evaluated based on the phenotypes of $V$. dahliaeinoculated plants using 3 disease grades: healthy, stunted or dead. 
The T3 plants of transgenic cotton were challenged by $V$. dahliae inoculation according to a previously described method (Zhang B. et al., 2012). The $V$. dahliae-inoculated cotton plants were scored and classified into five grades as described in our previous report (Zhang B. et al., 2012). The quantification of $V$. dahliae biomass was performed as described previously (Fradin et al., 2011).

\section{Expression Analysis of Defense-Related Genes in Transgenic Arabidopsis}

The expression levels of seven pathogenesis-related genes in Arabidopsis (PR1, PR2, PR3, PR5, PDF1.2, ERF1, and GST2) (Mazarei et al., 2007) were investigated 7 days after $V$. dahliae inoculation by qRT-PCR as described above with the $\beta$-tubulin gene as a reference (Hiratsu et al., 2003). The primers used for qRT-PCR were as follows: PR1-F (5' -gctcttgtaggtgctcttgttcttcct$\left.3^{\prime}\right)$ and PR1-R ( $5^{\prime}$-ctggttgtgaacccttagataatcttgtgg- $\left.3^{\prime}\right)$; PR2-F ( $5^{\prime}-\mathrm{c}$ aatctccettgctcgtgaatctctaccc- $\left.3^{\prime}\right)$ and PR2-R ( $5^{\prime}$-cgttatcaacagtggac tgggcgg- $\left.3^{\prime}\right)$; PR3-F ( $5^{\prime}$-ttaacggcctcctcgaagctgctattt- $\left.3^{\prime}\right)$ and PR3R ( $5^{\prime}$-cgcaacataaacagtgaaacatcattggaa- $\left.3^{\prime}\right)$; PR5-F ( $5^{\prime}$-caagaacgctt gcctgacgccta- $\left.3^{\prime}\right)$ and PR5-R (5'-gctccggtacaagtgaaggtgctcgtt- $\left.3^{\prime}\right)$; PDF1.2-F ( $5^{\prime}$-caagtgggacatggtcaggggtt- $\left.3^{\prime}\right)$ and PDF1.2-R ( $5^{\prime}$-cact tgtgtgctgggaagacatagttgc- $\left.3^{\prime}\right)$; ERF1-F ( $5^{\prime}$-agcagtccacgcaacaaaccta $\left.\mathrm{t}-3^{\prime}\right)$ and ERF1-R ( $5^{\prime}$-aaagcgactcttgaactctctcc- $\left.3^{\prime}\right)$; GST2-F ( $5^{\prime}$-cc agcttccgagaaggttcagtgagaa- $\left.3^{\prime}\right)$ and GST2-R ( $5^{\prime}$-gaaattgggcaatgag aaagccgctt- $\left.3^{\prime}\right)$; and $\beta$-tubulin-F ( $5^{\prime}$-cgtggatcacagcaatacagagcc- $\left.3^{\prime}\right)$ and $\beta$-tubulin-R ( $5^{\prime}$-cctcctgcacttccacttcgtcttc- $\left.3^{\prime}\right)$.

\section{Detection of Reactive Oxygen and Callose Formation in Transgenic Cotton}

Cotton roots were inoculated with a solution of $V$. dahliae $(1 \times$ $10^{7}$ conidia $/ \mathrm{ml}$ ) and incubated at $25^{\circ} \mathrm{C}$ in a humidified incubator for 5 days. Histochemical assays of $\mathrm{H}_{2} \mathrm{O}_{2}$ accumulation and callose deposition in infected roots were performed according to the method of Choi et al. (2012); the results were visualized with a fluorescence microscope under bright light and UV light, respectively.

\section{Tolerance of Arabidopsis Transgenic Plants to MeJA}

The seeds of WT and Gbvdr6-overexpressing lines were surfacesterilized with $70 \%$ ethanol for $30 \mathrm{~s}$ followed by exposure to $5.6 \% \mathrm{NaClO}$ for $5 \mathrm{~min}$; the seeds were then washed at least five times with sterile distilled water. The seeds were placed in $1 / 2 \mathrm{MS}$ medium with or without $20 \mu \mathrm{M}$ MeJA. Germination rates were calculated as the percentage of seeds with radicles protruding through the seed coat. The assays were replicated three times with 50 seeds each time. The root lengths of the seedlings (the distance to the root tip) were also measured using a ruler; at least 50 seeds were measured in each of three replicates (Gibson and Todd, 2015).

\section{Physiological Analysis of Gbvdr6-Overexpressing Plants}

Approximately $1 \mathrm{~g}$ of fresh root tissue from $V$. dahliae-inoculated or control plants was ground thoroughly in liquid nitrogen. The supernatant was used for analysis of PAL (EC 4.3.1.5) and CAT (EC 1.11.1.6) activity according to the instructions provided with the kit (Nanjing Jiancheng Bioengineering Institute). All enzyme activities are expressed as units $\mathrm{mg}^{-1}$ protein.

\section{Statistical Analysis}

All statistical analyses were performed using the "ANOVA analysis" software designed by the Nanjing Agricultural University. The Chi-square test and Fisher's exact test were used to evaluate the Verticillium wilt resistance of transgenic Gbvdr6 Arabidopsis and cotton, respectively. The Kruskal-Wallis test was conducted using the $\mathrm{R}$ language to analyze the expression of Gbvdr6 by the induction of $V$. dahliae, and Duncan's multiple range test was performed on the basis of the ANOVA analysis in SPSS 19.0 to compare the expression of Gbvdr6 in different organs and the expression of pathogenesis-related genes in transgenic Arabidopsis. In the graphs, asterisks and different letters indicate significant differences between treatments $\left({ }^{*} P<0.05\right.$; $\left.{ }^{* *} P<0.01\right)$, and letters shared in common between or among the experimental groups indicate no significant difference.

\section{RESULTS}

\section{Gbvdr6 Is Located in a Gene Cluster Adjacent to the Verticillium Wilt-Resistance QTL Hotspot}

Gbvdr6 cDNA consists of 3,328 bp (GenBank accession: KT809405), including an ORF of 3,204 bp that encodes a polypeptide comprising 1,067 amino acid (aa) residues with a calculated molecular weight of $119.28 \mathrm{kDa}$ and a predicted isoelectric point of 6.97 . The $3,328 \mathrm{bp}$ of cDNA sequence were mapped into the genomic region of 41706732-41710044 of the At_chr9 chromosome of upland cotton (G. hirsutum) cv. TM-1 (Li et al., 2015) with 99\% identity and a 3-bp gap (Figure S1), indicating that no intron exists in this gene. The sequences obtained by PCR amplification of the Hai7124 DNA template further confirmed that Gbvdr6 contains no intron. A BLASTX search of the non-redundant protein sequences database of NCBI revealed that the Gbvdr6 cDNA shared the highest identity (93\%) with a receptor-like protein in G. raimondii and with a series of receptor-like proteins associated with Verticillium wilt disease resistance in G. barbadense, Medicago truncatula, Humulus lupulus, Glycine max, Solanum torvum, and Solanum lycopersicoides. Phylogenetic analysis further demonstrated that Gbvdr6 is most closely related evolutionarily to cotton Verticillium wilt disease-resistance proteins (Figure 1A). SMART analysis indicated that Gbvdr6 possesses distinct domains corresponding to those of receptor-like proteins, including a signal peptide, multiple LRRs, a transmembrane domain and a cytoplasmic region (Figure 1B and Figure S1).

Sequence mapping through a BLASTN search of the genome of upland cotton (G. hirsutum) cv. TM-1 (Zhang et al., 2015) revealed that Gbvdr6 had two homologs in G. hirsutum. One was on the A01 (C1) chromosome, and the other was on the D01 (c15) chromosome. Similarly, GbVe1 and Gbvdr5 had homologs 
A

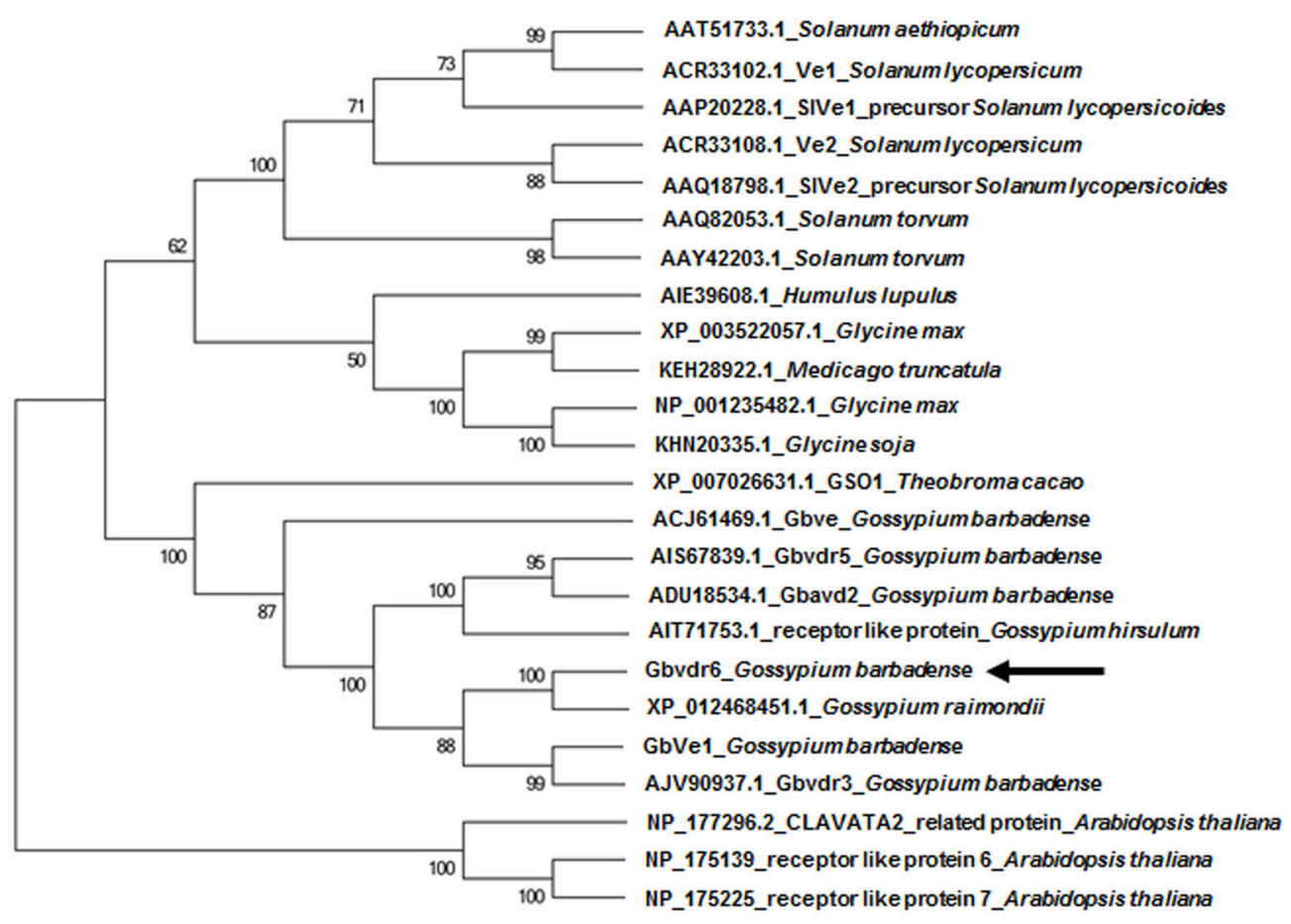

B

SP $\quad$ eLRR $\quad$ TM CD

N

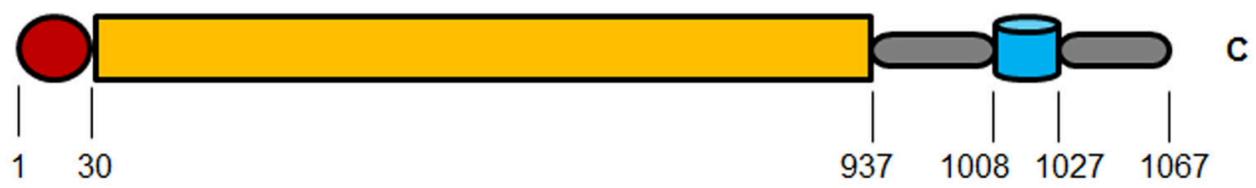

C

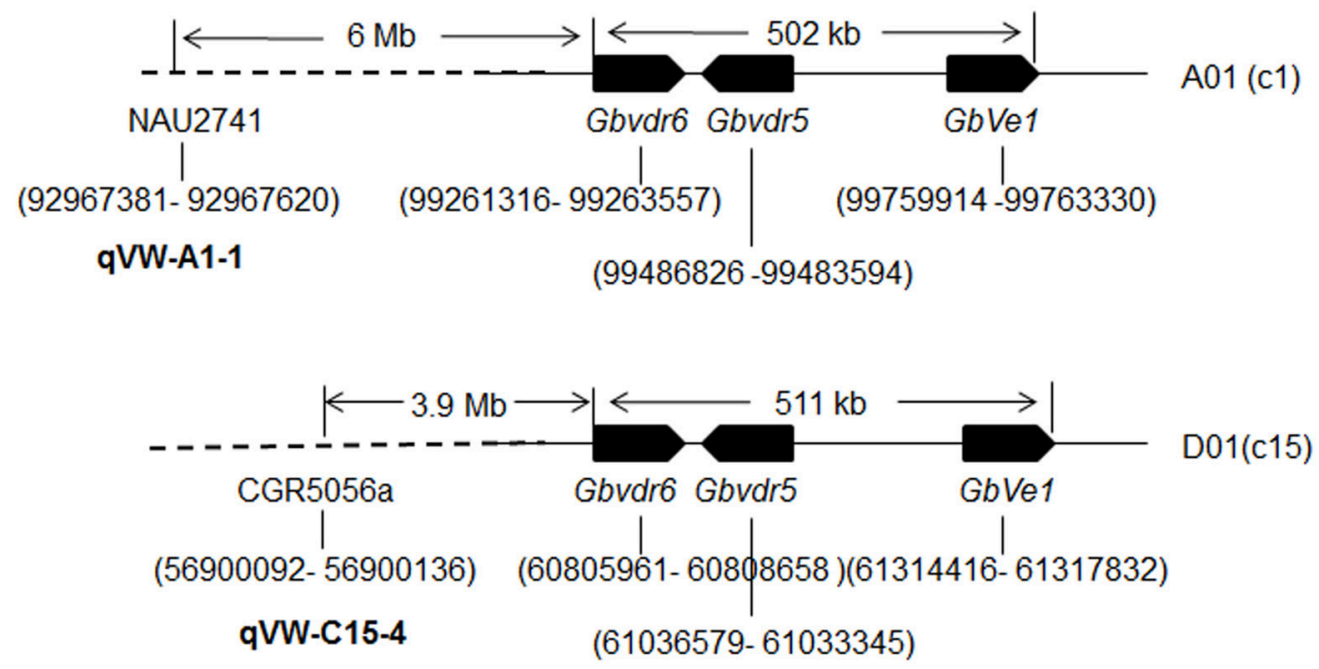

FIGURE 1 | Phylogenetic and structural analysis of Gbvdr6 gene. (A) Phylogenetic relationship of Gbvdr6 with other Ve-like proteins by MEGA6 software with the neighbor-joining (NJ) algorithm under 1,000 replicates of bootstrap. The numbers on the internal nodes are the percentage bootstrap support values. (B) Schematic diagram of Gbvdr6 protein domain architecture showing signal peptide (SP) at N-terminus, followed by extracellular leucine-rich repeat (eLRRs), transmembrane (TM) domain and cytoplasmic domain (CD) at C-terminus. The numbers indicate the domain regions. (C) Schematic diagram of physical locations of Gbvdr6, Gbvdr5, and GbVe1 and the SSR markers flanking the known Verticillium wilt-resistant QTLs in the chromosomes of tetraploid cotton. The numbers in brackets indicate the physical positions in the chromosome. NAU2741 and CGR5056a are the SSR markers that flank Verticillium wilt resistance QTLs qWW-A1-1 and qVW-C15-4 in the A01(c1) and D01(c15) chromosomes, respectively. 


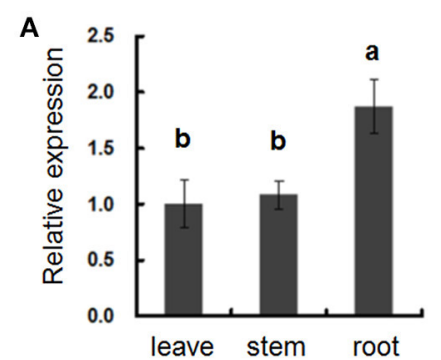

B

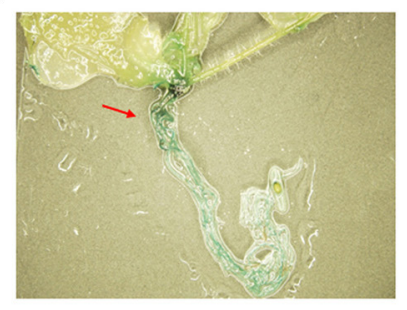

C
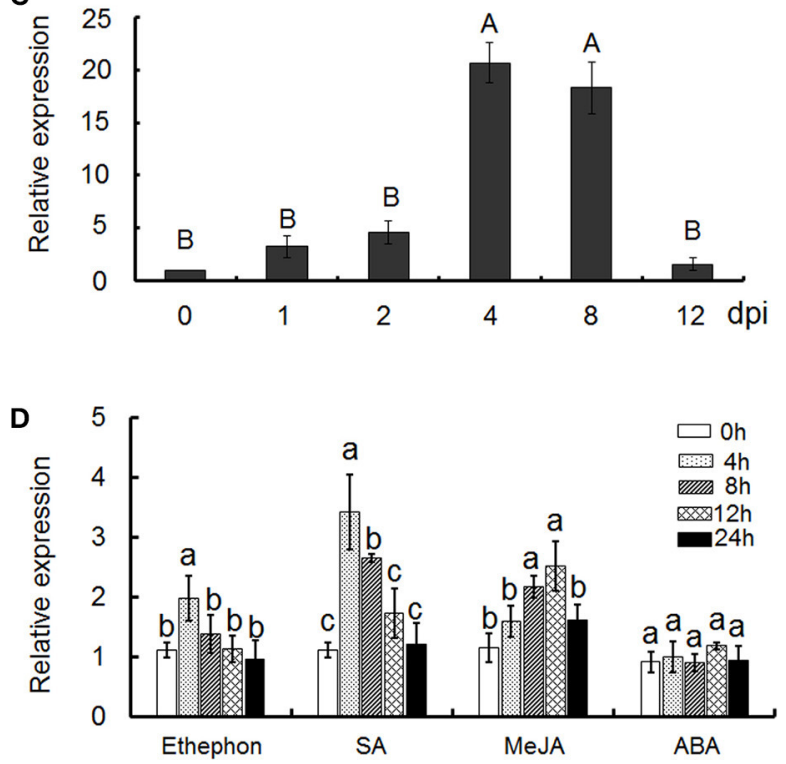

FIGURE 2 | Expression pattern analysis of the Gbvdr6 gene. (A) The transcript levels of Gbvdr6 in different tissues of Hai7124. Values were expressed as fold changes of transcript levels in the different tissues with respect to that of leaves with the $2^{-\Delta \Delta C T}$ Method. Error bars represented SE of three biological replicates. Duncan's multiple range test was conducted, and the different letters in graphs indicate significant differences between treatments $(P<0.05)$. (B) Gus activities in transgenic Arabidopsis plantlets containing the pGbvdr6: Gus construct. (C) The expression patterns of Gbvdr6 in response to infection by V. dahliae. The transcript levels of Gbvdr6 were measured by real-time reverse-transcription PCR with the UBQ14 gene as the internal control. Values were expressed as fold changes of transcript levels in the $V$. dahliae inoculated samples at fixed point of time with respect to that in non-inoculated samples at 0 dpi with the $2^{-\Delta \Delta C T}$ Method. Error bars represented SE of three biological replicates. Kruskal-Wallis test was conducted, and the different letters in graphs indicate significant differences between treatments $(P<0.01)$. (D) The expression patterns of Gbvdr6 in response to phytohormones. Values were expressed as fold changes of transcript levels in the phytohormones treated root samples at fixed point of time with respect to the transcript levels in the mock samples. Error bars represented SE of three biological replicates and the different letters in graphs indicate significant differences after treatments $(P<0.05)$.

on the A01 (C1) and D01 (c15) chromosomes of G. hirsutum. The physical positions of these cotton RLPs further revealed that the homologs of Gbvdr6 were physically clustered with those of GbVe1 and Gbvdr5 on the A01 or D01 chromosome of upland cotton (Figure 1C). At the same time, one Verticillium wiltresistance QTL hotspot, C15-VW-QTL-rich-1, including qVWC15-2, qVW-C15-3, and qVW-C15-4, and four QTLs including qVW-A1-1, qRV07DF2-A1-1, qDL52T2-c15, and qRD8092-D11 were detected on the A01 (C1) and D01 (c15) chromosomes of tetraploid cotton, respectively (Ning et al., 2013; Fang et al., 2014; Wang et al., 2014; Shi et al., 2016). The flanked markers of these QTLs were anchored to the TM-1 genome through BLASTN. The physical positions of these markers on the chromosome further revealed that the SSR marker CGR5056a, which was linked to qVW-C15-4, was approximately 3.9 Mb from the gene cluster of Gbvdr6-Gbvdr5-GbVe1 homologs on the D01 (C15) chromosome, whereas the SSR marker NAU2741 that flanked qVW-A1-1 was only $6 \mathrm{Mb}$ upstream of the gene cluster on the A01 (C1) chromosome (Figure 1C).

\section{Gbvdr6 Is Highly Expressed in Root and Is Induced by V. dahliae, SA, MeJA, and ETH Treatment}

The expression patterns of Gbvdr6 in various cotton tissues were examined by quantitative real-time PCR (qRT-PCR) analysis.
Gbvdr6 was ubiquitously expressed in all tested tissues, and its transcripts accumulated to the highest level in root (Figure 2A). Gbvdr6 promoter-driving GUS activity was also found mostly in the root of transgenic Arabidopsis (Figure 2B), consistent with the results of qRT-PCR. To further investigate the involvement of Gbvdr6 in the response to $V$. dahliae infection, gene expression was analyzed after inoculation of cotton seedlings with $V$. dahliae. The expression of Gbvdr6 was induced until 4 days postinoculation (dpi), reached maximum levels at 4-8 dpi, and then returned to the control levels at 12 dpi (Figure 2C). SA, MeJA, and ETH but not ABA markedly increased Gbvdr6 transcript levels in roots and the transcript levels increased during the early stages of treatment and decreased thereafter (Figure 2D).

\section{Gbvdr6 Protein Is Localized on the Plasma Membrane}

To investigate the subcellular localization of Gbvdr6, Gbvdr6 was fused with to the N-terminus of GFP and transiently co-expressed with the plasma membrane marker mCherry in $N$. benthamiana leaves. Confocal microscopy revealed that the green fluorescent signal from the Gbvdr6-GFP fusion and the red fluorescent signal from mCherry were co-localized (Figure 3), indicating that Gbvdr6 is localized on the plasma membrane. 


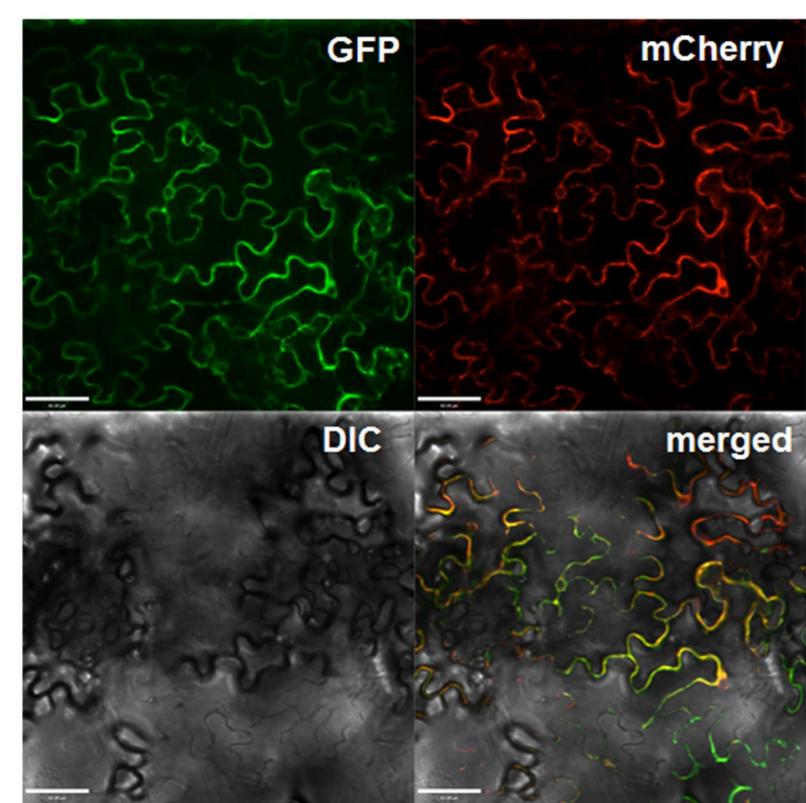

FIGURE 3 | Subcellular localization of Gbvdr6 in epidermal cells of N. tabacum leaves. The Gbvdr6-GFP fusion was transiently co-expressed with the plasma membrane marker mCherry. The images were taken under a confocal microscope at $48 \mathrm{~h}$ after agro-infiltration. GFP: fluorescence of Gbvdr6-GFP fusion, mCherry: fluorescence of the plasma membrane marker mCherry, DIC, differential interference contrast; merged, a merged image. Scale bar $=60 \mu \mathrm{m}$.

\section{Overexpression of Gbvdr6 Enhances Resistance to Verticillium in Arabidopsis}

To explore the function of Gbvdr6, an overexpression cassette in the background of the binary vector pCAMBIA2301 under the control of the CaMV35S promoter was introduced into the Arabidopsis thaliana genome. The transgenic plants were screened on kanamycin plates and confirmed by semiquantitative RT-PCR (Figure 4A). The T3 transgenic Arabidopsis lines were challenged with $V$. dahliae. At $28 \mathrm{dpi}$, the healthy, stunted and dead phenotypes of Arabidopsis plants in response to $V$. dahliae infection were scored. Overexpression of $G b v d r 6$ in transgenic Arabidopsis significantly improved resistance to $V$. dahliae, the death rate of $46.7 \%$ observed in wild-type Arabidopsis plantlets was reduced to $3.3 \%$ in the transgenic plants (Figure 4B). However, transgenic Gbvdr6 Arabidopsis was not immune to $V$. dahliae, and its growth was stunted (Figure 4C).

\section{Overexpression of Gbvdr6 Significantly Activates Defense Genes in Arabidopsis}

The expression levels of $P R 1, P R 2$, and $P R 5$, which are marker genes for SA signaling, those of PDF1.2 and ERF1, genes involved in ET- and JA-signaling and that of GST2, a gene involved in ET signaling, were investigated by qRT-PCR. After mock inoculation, the selected pathogenesis-related genes $P R 1, P R 2$, $P R 5, P R 3, E R F 1$, and PDF1.2 were significantly upregulated in Gbvdr6-overexpressing Arabidopsis (Figure 5). The expression
A

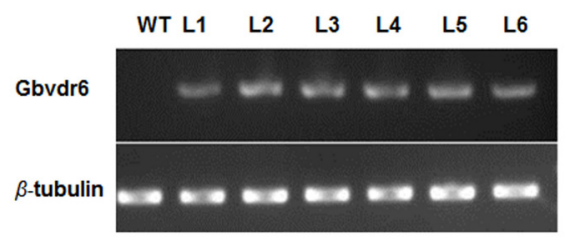

B

C

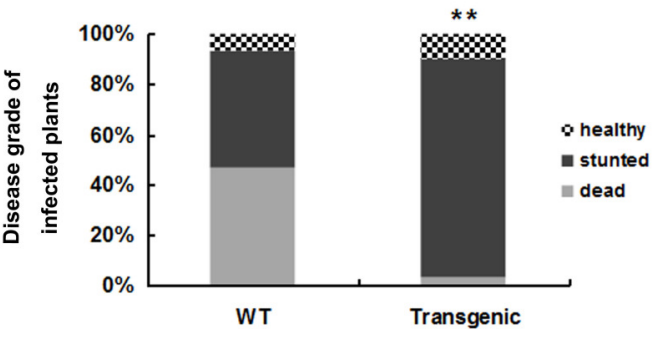

WT

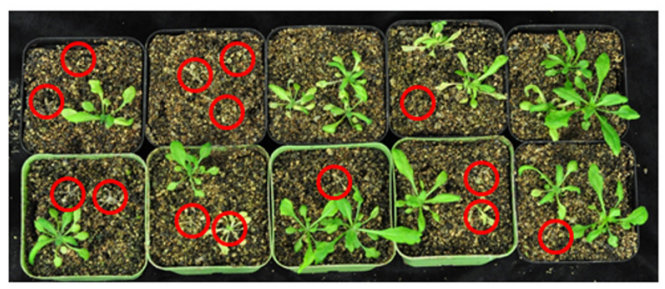

Transgenic

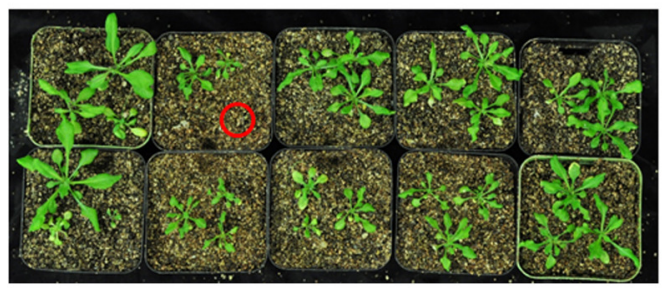

FIGURE 4 | Gbvdr6 over-expressed Arabidopsis enhanced resistance to V. dahliae. (A) The semi-quantitative RT-PCR of Gbvdr6 over-expressed Arabidopsis. The $\beta$-tubulin was the internal control, and L1-L6 are the transgenic Arabidopsis lines. (B) The numbers of healthy, stunted and dead Arabidopsis plants were scored and statistically analyzed. Thirty plantlets were tested in each line. The experiment was conducted twice with similar results. The chi-squared test is used to determine whether there is a significant difference. The asterisk indicated above the columns means ${ }^{\star \star} P<0.01$. (C) The phenotype of the transgenic and non-transgenic cotton induced by Verticillium dahliae. The photos were taken at 28 days post-inoculation with $V$. dahliae. The red circles indicated the dead plants.

levels of PR1, PR2, PR5, PR3, ERF1, and PDF1.2 were further elevated in plants after $V$. dahliae infection. In contrast, the ET signaling marker gene GST2 was not affected by overexpression of Gbvdr6 or by infection with $V$. dahliae. The transcript abundances of $P R 1, P R 2, P R 3$, and PR5 in the $V$. dahliaeinfected transgenic plants were approximately 2.5-, 6-, 18and 4.5 -fold, respectively, those of the infected control plants. The transcript abundances of ERF1 and PDF1.2 increased approximately 2 - and 5-fold, respectively, in the $V$. dahliaeinfected transgenic plants compared to the infected control plants. These results indicate that overexpression of Gbvdr6 significantly activates the expression of defense genes and suggests that it might be involved in the SA and JA/ET-mediated signaling pathways. 


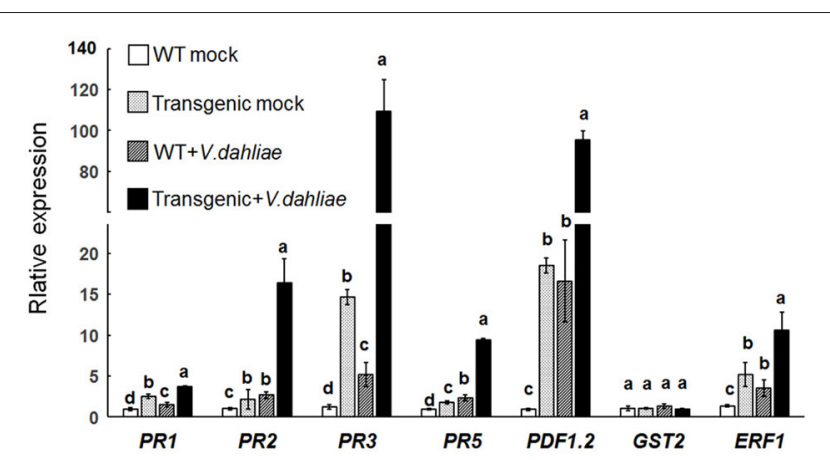

FIGURE 5 | Upregulation of pathogenesis-related genes in the Gbvdr6 over-expressed Arabidopsis. The expression levels of marker genes PR1, PR2, and PR5 in SA signaling, PR3, PDF1.2, and ERF1 in ET/JA signaling, and GST2 in ET signaling were measured by real-time reverse-transcription PCR with $\beta$-tubulin gene as the internal control. Data were the means $\pm \mathrm{SE}$ of three biological replicates. Duncan's multiple range test was carried out within genes, and different letters in the graphs indicate significant differences between treatments $(P<0.05)$.
45.2 (Figure 7D). The fungal biomass present in the V. dahliaeinfected plants was further analyzed by qPCR. The biomass of Verticillium was approximately 8 -fold higher in the wild-type plants than in the L1 plants at $30 \mathrm{dpi}$ (Figure 7E), confirming that Gbvdr6 overexpression confers resistance to Verticillium in cotton. In addition, the activities of catalase (CAT) and phenylalanine ammonia lyase (PAL) were measured; the activities of the two enzymes in wild-type and L1 transgenic plants showed no difference before infection. The activity of these enzymes was much higher in the transgenic line L1 than in the wild-type strain at 1-7 days after infection (Figure 7F).

The accumulation of reactive oxygen species (ROS) in the host during the early stage of pathogen infection is helpful in fighting against pathogens and in activating cellular programs that help the plant cope with pathogens. At 5 days post-inoculation (dpi) with $V$. dahliae, the roots of Gbvdr6-overexpressing cotton accumulated significantly higher levels of $\mathrm{H}_{2} \mathrm{O}_{2}$ than those of WT plants (Figure 8, upper). Callose deposition is another immune response to pathogen challenge. At $5 \mathrm{dpi}$, the roots of Gbvdr6-overexpressing cotton deposited more callose at the site of infection than those of wild-type Arabidopsis (Figure 8, lower).

\section{DISCUSSION}

We used the genome walking method to clone the full-length sequence of Gbvdr6 from G. barbadense Hai7124 cDNA and genomic DNA. Sequence analysis of Gbvdr6 cDNA and DNA revealed no introns within this gene, similar to the reported Verticillium wilt-resistance genes $V e 1$ in tomato and GbVe, GbVe1, and Gbvdr5 in G. barbadense (Kawchuk et al., 2001; Zhang et al., 2011; Zhang B. et al., 2012; Yang et al., 2015a). The release of the genome sequence of upland cotton (G. hirsutum) cv. TM-1 (Li et al., 2015) made it possible for us to compare the Gbvdr6 gene from G. barbadense Hai7124 with that of a Verticillium wilt-sensitive strain. Twenty-two nucleotides of the Gbvdr6 gene sequence and seventeen amino acids of the Gbvdr6 protein differed between the resistant strain $\mathrm{cv}$. Hai7124 and the susceptible strain cv.TM-1, suggesting that the three-nucleotide deletion in Gbvdr6 of Hai7124 did not affect its translation (Figure S1). Single-nucleotide polymorphisms between the resistant and susceptible cultivars have also been detected in cotton GbVe and Gbvdr5 (Zhang et al., 2011; Yang et al., 2015a) and tomato Ve1 (Fradin et al., 2009). However, the amino acid sequences of GbVe in the resistant cv. Pima 9053 and GhVe in the susceptible cv. CRI8 are identical (Zhang et al., 2011). In contrast, compared with Gbvdr5 from the resistant cv. Hai7124, a single-nucleotide deletion that results in termination of translation was found in Ghvdr5 from the susceptible cotton genotype Yuman1 (Yang et al., 2015a). The $V e 1$ sequence also differs in resistant and susceptible tomato cultivars, with a single nucleotide deletion accompanied by premature termination in susceptible tomato genotypes (Fradin et al., 2009). Multiple alignment analysis further showed that the Gbvdr6 protein shared high homology with other Ve1homologous RLPs in cotton. These cotton Ve1 homologs possess similar domains, such as a signal peptide domain, multiple 
A

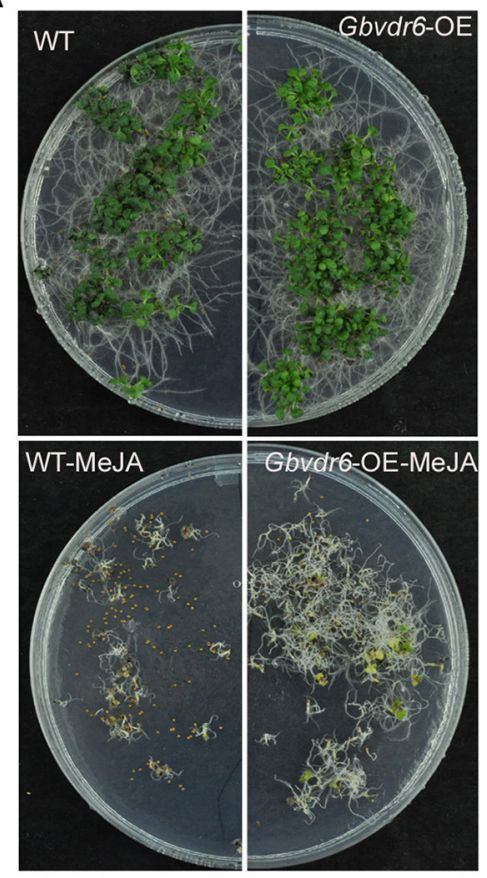

B

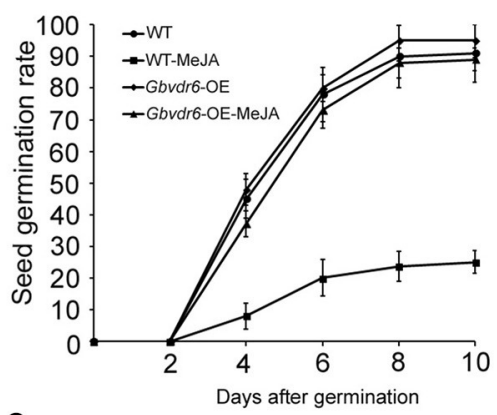

C

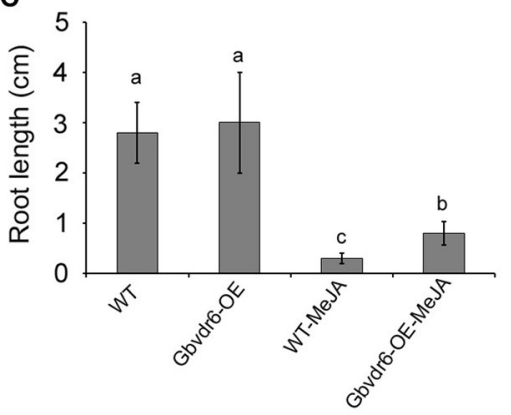

FIGURE 6 | Gbvdr6 over-expressed Arabidopsis is more insensitive to MeJA compared with the wild type. (A) The phenotype of Gbvdr6 over-expressed Arabidopsis (Gbvdr6-OE) and wild type after MeJA treatment. The WT and Gbvdr6-OE are the seedlings grown on the plate without MeJA, and the WT-MeJA and Gbvdr6-OE-MeJA indicates the seedling on the plate with 20 um MeJA. The photos were taken at 30 days after sowing. (B) Assay of seed germination rate of Gbvdr6 over-expressed Arabidopsis (Gbvdr6-OE) and wild type in the presence of exogenous MeJA. Germination rates of the seeds were analyzed at the indicated time points. The data represent means \pm SD of three independent replicates with at least 50 seeds counted per replicate. (C) Assay of root length of Gbvdr6 over-expressed Arabidopsis (Gbvdr6-OE) and wild type in the presence of exogenous MeJA at 30 days after sowing. Significant difference between different lines is indicated by different letters $(P<0.05)$.

eLRRs, a transmembrane domain and a cytoplasmic tail. These characterized domains, particularly the eLRRs, appear to provide a structural framework for functionality in Verticillium wilt resistance (Zhang et al., 2011). Recent studies of the functional determinants of Ve1 through domain swapping and site-directed mutagenesis have revealed that three consecutive eLRR regions (i.e., eLRR1-eLRR8, eLRR20-eLRR23, and eLRR32-eLRR37), the non-LRR island domain (C2), the transmembrane region and the cytoplasmic tail are critical for Vel functionality (Fradin et al., 2014; Zhang Z. et al., 2014). However, the Gbvdr6 domains and the regions responsible for Verticillium wilt resistance remain to be further investigated.

$V$. dahliae is a soil-borne pathogen that can successfully penetrate the root epidermis to invade the cortical tissues and cause systemic infection in plants (Klosterman et al., 2009). Analysis of the expression of Gbvdr6 in various tissues revealed that the highest expression occurs in cotton root, which may allow the plant to set up a protective barrier against infection by $V$. dahliae. The locations of proteins are critically linked to their functionality. In this study, Gbvdr6 was found to be localized to the plasma membrane, as expected. This is consistent with the results of previous studies in which the proteins encoded by Verticillium wilt-resistance genes such as Ve1, GbVe, and Gbvdr5 were all shown to be localized to the plasma membrane (Zhang et al., 2011; Fradin et al., 2014; Yang et al., 2015a).
The strong homology between Gbvdr6 and the reported genes encoding cotton RLPs, including GbVe (Zhang et al., 2011), GbVe1 (Zhang B. et al., 2012), and Gbvdr5 (Yang et al., 2015a), enabled us to investigate their physical locations. Notably, $G b v d r 6, G b V e 1$ and $G b v d r 5$ were found to be physically clustered on the same chromosome. Previous studies have shown that $C f$ genes are highly homologous and are generally located in clusters (Kruijt et al., 2005). A few $c f$ homologs have protective functions against C. fulvum, whereas others may serve as a reservoir of novel $C f$ genes through sequence exchange between homologs (Kruijt et al., 2005). Dozens of Verticillium wilt-resistance QTLs have been detected in most of the 26 tetraploid cotton chromosomes of various cotton populations (Zhang J. et al., 2014). This enabled us to investigate whether Gbvdr6 is related to the known Verticillium wilt- resistance QTLs. Interestingly, a Verticillium wilt-resistance QTL hotspot was identified adjacent to a gene cluster with homology to Gbvdr6-Gbvdr5-GbVe1 in the A01 (C1) and D01 (c15) chromosomes of tetraploid cotton, supporting the idea that this gene cluster is vital to Verticillium wilt resistance.

Transcriptome profiling of G. barbadense inoculated with $V$. dahliae revealed large accumulations of defense-related transcripts, including transcripts of the $P R 1, P R 2, P R 3, P R 5$, and $B A G$-like genes and transcripts encoding dynamin-related proteins (Zhang et al., 2013), suggesting a role in resistance 

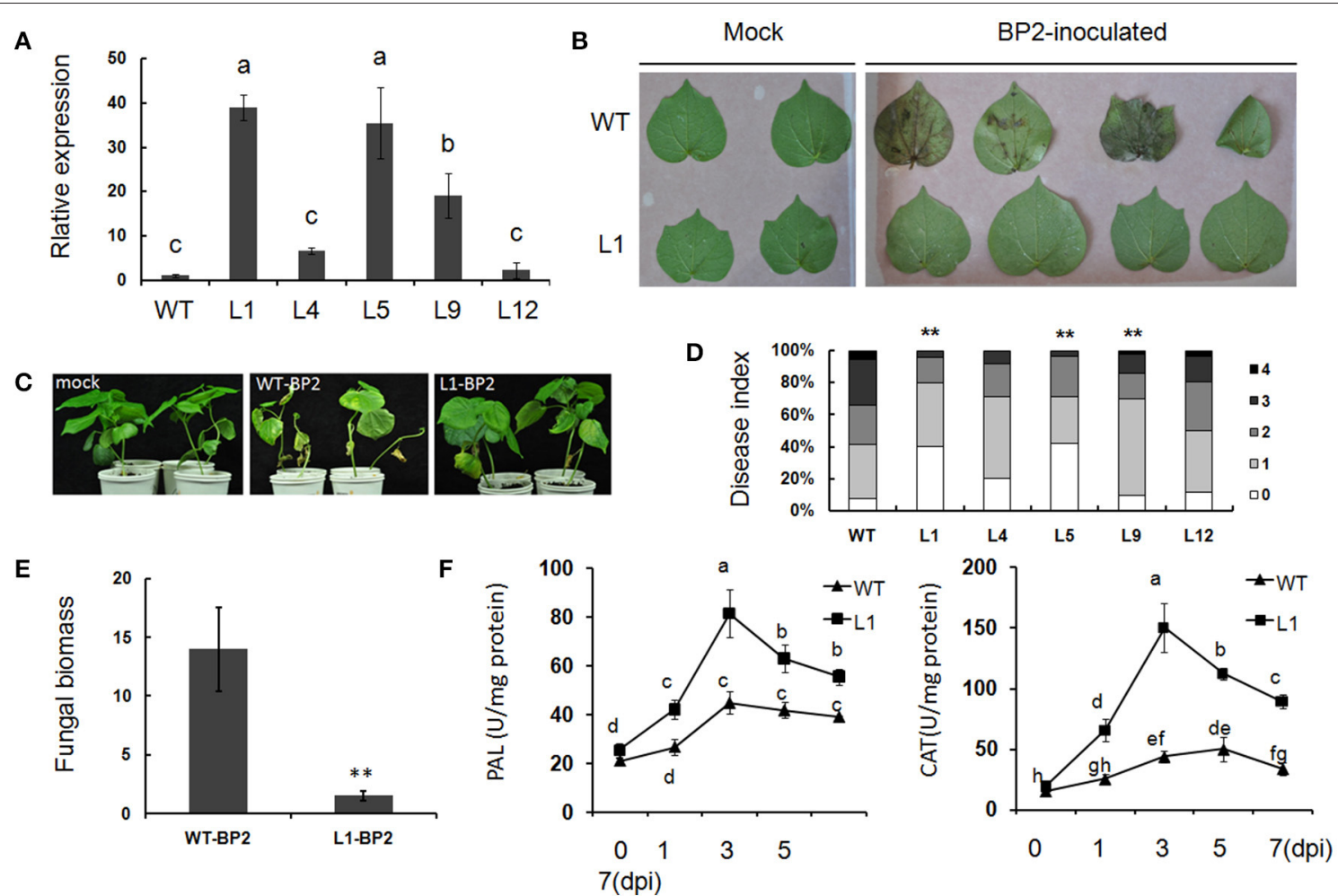

FIGURE 7 | Gbvdr6 over-expressed cotton enhanced resistance to V. dahliae. (A) Varied expressional levels of Gbvdr6 in the transformed plants. Gbvdr6 relative expressional levels of the T3 generation were measured by qRT-PCR and calculated in relation to the wild type plants according to the $\triangle \triangle \mathrm{Ct}$ method with the $U B Q 14$ gene as the internal control. L1, L4, L5, L9, and L12 are the transgenic cotton lines. Different letters on the bars designate statistically significant differences $(P<0.05)$ according to Duncan's multiple range test. (B) Leaves of Gbvdr6 over-expressed cotton (L1) and wild type inoculated with $V$. dahliae in vitro. The photos were taken at 5 days after inoculation. (C) The Gbvdr6 over-expressed cotton inoculated with V. dahliae in vivo. The photos were taken at 15 days after inoculation. (D) Assay of disease index of transgenic lines and wild type by the $V$. dahliae isolate BP2. The degree of disease was divided into five grades with disease scores ranging between 0 and 4 , and fisher's exact test was conducted to determine whether there is a significant difference between the WT and transgenic lines. The asterisk indicated above the columns means ${ }^{\star \star} P<0.01$. (E) Fungal biomass upon inoculation with $V$. dahliae isolate BP2. It was determined by qRT-PCR, and the bars represent Verticillium ITS transcript levels relative to the cotton UBQ14 gene. Data were the means \pm SE of three biological replicates and significant differences by Student's test for $P<0.01$ are indicated by double asterisks. (F) Enzyme activity of PAL and CAT of the Gbvdr6 over-expressed and WT plants after the inoculation of $V$. dahliae isolate BP2. Duncan's multiple range test was conducted, and the different letters in graphs indicate significant differences $(P<0.05)$.

to $V$. dahliae. Overexpression of Gbvdr6 in Arabidopsis also enhanced the expression of the pathogenesis-related genes PR1, PR2, PR5, PR3, ERF1, and PDF1.2, and these genes were further upregulated when transgenic Gbvdr6 Arabidopsis was subjected to $V$. dahliae infection. Because $P R 1, P R 2$ and $P R 5$ are marker genes for SA signaling and PR3, ERF1, and PDF1.2 are marker genes for JA/ET signaling (Mazarei et al., 2007), the findings suggest that Gbvdr6 plays a role in activating both SA signaling and JA/ET signaling. In contrast, the expression of the marker gene GST2 in ET signaling was similar in transgenic and WT plants, even when the plants were infected with. $V$. dahliae. This finding is inconsistent with previous results obtained in Gbvdr5- and GbVe1-overexpressing transgenic Arabidopsis, in which GST1 was significantly more upregulated in the transgenic than in the WT plants after V. dahliae infection (Zhang B. et al., 2012; Yang et al., 2015a).

The relationship between SA and JA is not always antagonistic and may sometimes be collaborative (Mur et al., 2006; Liu et al., 2016). Moreover, enhanced JA levels concomitant with increased
SA production and heightened expression of SA-responsive $P R$ genes have been found under some conditions (Thaler et al., 2012; Tong et al., 2012). A noncanonical mechanism has been reported in which the JA signaling pathway can be activated following SA accumulation and through the SA receptors NPR3 and NPR4 (Liu et al., 2016). In addition, Verticillium wilt is a kind of hemibiotrophic pathogen that colonizes its hosts as a biotroph during the first part of its life cycle in the xylem, killing them during the subsequent necrotrophic phase (Klosterman et al., 2011). The SA- and JA-dependent signaling pathways are thought to interact synergistically during plants' responses to hemibiotrophic pathogens (Edgar et al., 2006; Pan et al., 2014; Yi et al., 2014; Zhu et al., 2017). The secreted isochorismatase VdIsc1 contributes to the full pathogenesis of Verticillium dahliae by impairing SA synthesis (Liu et al., 2014). Plants infected with wild-type $V$. dahliae isolates accumulate more free JA and SA than those infected with the icsh1 mutant (Zhu et al., 2017). VdSCP7 of $V$. dahliae can activate both SA and JA signaling, and such signaling alters the plants' susceptibility 

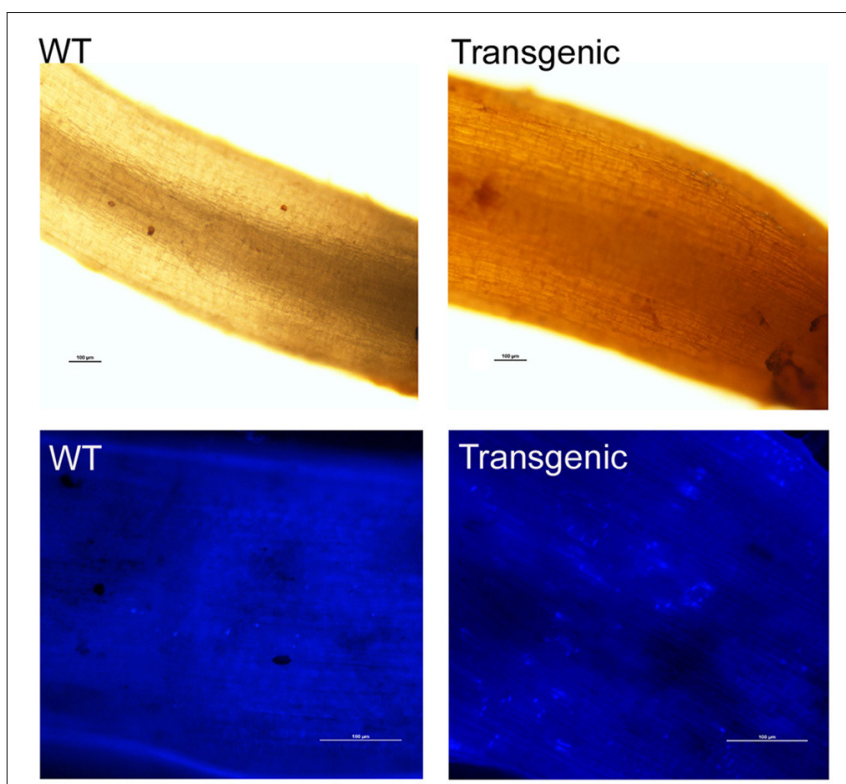

FIGURE 8 | Hydrogen peroxide and callose accumulation in the Gbvdr6 over-expressed cotton and WT plant in response to $V$. dahliae. Roots from the transgenic and the WT at 5 days post-inoculation with $V$. dahliae were stained with 3,3'-diaminobenzidinetetrahydrochloride (DAB), and photos were taken under a fluorescence microscope with bright light. Scale bar $=100 \mu \mathrm{m}$ (upper). Callose accumulation (lower). Roots from the transgenic and the WT at 5 days post-inoculation with $V$. dahliae were stained with aniline blue, and photos were taken under a fluorescence microscope with UV light. Scale bar $=100 \mu \mathrm{m}$.

to Botrytis cinerea and Phytophthora capsici (Zhang et al., 2017).

Unlike wild-type plants, the Gbvdr6-overexpressing plants in our study were not sensitive to MeJA, suggesting that the JA signaling pathway in these plants may be blocked; however, JAsensitive genes were more highly expressed in the transgenic plants. These facts seem contradictory and puzzling. The JA signaling pathway is complex, and some mutants, such as coil and jin1, both of which are insensitive to MeJA, show discrepancies. However, PDF1.2 was observed to be induced much more strongly in jin1-1 plants throughout the infection process, whereas it was repressed in coil plants. In addition, JIN1 has been shown to negatively regulate the expression of PDF1.2 in response to MeJA treatment (Boter et al., 2004; Lorenzo et al., 2004; Laurie-Berry et al., 2006). The Gbvdr6overexpressing plants were insensitive to JA but expressed high levels of PDF1.2, suggesting that a mediator such as JIN1 may be repressed and that this repression may be involved in the resistance mediated by Gbvdr6. In addition, the Arabidopsis mutant cev1 has constitutively active JA/ET signal pathways but is insensitive to JA, as shown by the fact that its morphological traits were unchanged when treated with JA (Ellis and Turner, 2001).

In response to pathogens, plants have evolved a series of inducible responses including $\mathrm{HR}$, the production of ROS and callose, and the production and accumulation of PR proteins, phytoalexins, and antimicrobial proteins (Luo et al., 2014). Ve1mediated Verticillium resistance was activated by the $V$. dahliae effector protein Ave1 and triggered an HR in tomato (Jonge et al., 2012). However, Ve1-mediated HR may be determined by the plant species because no HR was found in N. benthamiana or Arabidopsis, suggesting that the HR is not absolutely required for Verticillium wilt resistance (Zhang et al., 2013a,b). Moreover, $V e 1$-mediated resistance involved the induction of ROS and increased the activities of peroxidase, phenylalanine ammonia lyase, and lignins (Gayoso et al., 2010). The significant induction of ROS in Gbvdr6-overexpressing Arabidopsis at the early time points of Verticillium infection is consistent with the findings for $V e 1$. The rapid increase in ROS levels observed in transgenic Gbvdr6 Arabidopsis plants is likely related to several of the plant's known defense functions, including the production of a compound that is toxic to microbes and the participation of ROS in cell wall reinforcement, lipid peroxidation, signal transduction cascades and the triggering of defensive responses (Gayoso et al., 2010). Callose deposition facilitates the host's defenses against pathogen penetration at early time points of infection (Blümke et al., 2013; Ellinger et al., 2013). The deposition of higher amounts of callose in Gbvdr6-overexpressing cotton roots at 5 days post-inoculation suggested increased resistance to $V$. dahliae penetration. This finding is consistent with previously reported findings for $G b v d r 5$, the overexpression of which caused more callose to be deposited at the site of infection (Yang et al., 2015a).

Our observations suggest that Gbvdr6 contributes to Verticillium wilt resistance. Gbvdr6 is a receptor-like protein located on the plasma membrane and has been suggested to detect extracellular microbe-derived ligands. Receptor-like proteins are hypothesized to interact with receptor-like kinases (RLKs) or other membrane proteins to activate downstream signaling (Jeong et al., 1999; Humphries et al., 2011; Yang et al., 2012; Postma et al., 2016). However, although the increased survival rate of Gbvdr6-overexpressing Arabidopsis plantlets after $V$. dahliae infection suggests a role of Gbvdr6 in defense against pathogens, the transgenic plants were not immune to this pathogen, unlike transgenic Ve1 Arabidopsis, which was immune to infection by the $V$. dahliae isolate racel (Fradin et al., 2011). The overexpression of Gbvdr6 in Arabidopsis activated marker genes for SA signaling and JA/ET signaling and triggered ROS production and callose deposition at early time points after infection in transgenic cotton. Gbvdr6 was physically clustered with GbVe1 and Gbvdr5, another two Verticillium wilt-resistance genes on the same chromosomes in tetraploid cotton. However, the relationships between these RLP gene clusters and their nearby Verticillium wiltresistance QTLs on the same chromosomes remain to be further explored.

\section{AUTHOR CONTRIBUTIONS}

YY and XL performed the laboratory experiments and analyzed data, TC performed bioinformatics analyses. All authors contributed to experimental design and conceived experiments. 
YY and ZM wrote the manuscript and designed figures and schematics.

\section{FUNDING}

This work was supported by the National Natural Science Foundation of China [grant No. 31672096]; Jiangsu Agricultural Science and Technology Innovation Fund (CX(14)2007); Outstanding youth fund of Jiangsu Province (BK20160016); The National Key Research and Development Program of China

\section{REFERENCES}

Artico, S., Nardeli, S. M., Brilhante, O., Grossi-de-Sa, M. F., and Alves-Ferreira, M. (2010). Identification and evaluation of new reference genes in Gossypium hirsutum for accurate normalization of real-time quantitative RT-PCR data. BMC Plant Biol. 10:49. doi: 10.1186/1471-2229-10-49

Bent, A. (2006). Arabidopsis thaliana floral dip transformation method. Methods Mol. Biol. 343, 87-103. doi: 10.1385/1-59745-130-4:87

Blümke, A., Somerville, S. C., and Voigt, C. A. (2013). Transient expression of the Arabidopsis thaliana callose synthase PMR4 increases penetration resistance to powdery mildew in barley. Adv. Biosci. Biotechnol. 2013, 810-813. doi: 10.4236/abb.2013.48106

Boter, M., Ruiz-Rivero, O., Abdeen, A., and Prat, S. (2004). Conserved MYC transcription factors play a key role in jasmonate signaling both in tomato and Arabidopsis. Genes Dev. 18, 1577-1591. doi: 10.1101/gad.297704

Camacho-Cristóbal, J. J., Martin-Rejano, E. M., Herrera-Rodriguez, M. B., Navarro-Gochicoa, M. T., Rexach, J., and Gonzalez-Fontes, A. (2015). Boron deficiency inhibits root cell elongation via an ethylene/auxin/ROSdependent pathway in Arabidopsis seedlings. J. Exp. Bot. 66, 3831-3840. doi: $10.1093 /$ jxb/erv186

Chen, L., Jiang, B., Wu, C., Sun, S., Hou, W., and Han, T. (2014). GmPRP2 promoter drives root-preferential expression in transgenic Arabidopsis and soybean hairy roots. BMC Plant Biol. 14:245. doi: 10.1186/s12870-014-0245-z

Cheng, H. Q., Han, L. B., Yang, C. L., Wu, X. M., Zhong, N. Q., Wu, J. H., et al. (2016). The cotton MYB108 forms a positive feedback regulation loop with CML11 and participates in the defense response against Verticillium dahliae infection. J. Exp. Bot. 67, 1935-1950. doi: 10.1093/jxb/erw016

Choi, D. S., Hwang, I. S., and Hwang, B. K. (2012). Requirement of the cytosolic interaction between pathogenesis-related protein 10 and leucine-rich repeat protein 1 for cell death and defense signaling in pepper. Plant Cell 24, 1675-1690. doi: 10.1105/tpc.112.095869

Duan, X., Zhang, Z., Wang, J., and Zuo, K. (2016). Characterization of a novel cotton subtilase gene GbSBT1 in response to extracellular stimulations and its role in verticillium resistance. PLOS ONE 11:e0153988. doi: 10.1371/journal.pone.0153988

Edgar, C. I., McGrath, K. C., Dombrecht, B., Manners, J. M., Maclean, D. C., Schenk, P. M., et al. (2006). Salicylic acid mediates resistance to the vascular wilt pathogen Fusarium oxysporum in the model host Arabidopsis thaliana. Australas. Plant Pathol. 35, 581-591. doi: 10.1071/AP06060

Ellinger, D., Naumann, M., Falter, C., Zwikowics, C., Jamrow, T., Manisseri, C., et al. (2013). Elevated early callose deposition results in complete penetration resistance to powdery mildew in Arabidopsis. Plant Physiol. 161, 1433-1444. doi: 10.1104/pp.112.211011

Ellis, C., and Turner, J. G. (2001). The Arabidopsis mutant cev1 has constitutively active jasmonate and ethylene signal pathways and enhanced resistance to pathogens. Plant Cell 13, 1025-1033. doi: 10.1105/tpc.13.5.1025

Fang, H., Zhou, H., Sanogo, S., Flynn, R., Percy, R. G., Hughs, S. E., et al. (2013). Quantitative trait locus mapping for Verticillium wilt resistance in a backcross inbred line population of cotton (Gossypium hirsutum $\times$ Gossypium barbadense) based on RGA-AFLP analysis. Euphytica 194, 79-91. doi: 10.1007/s10681-013-0965-4

Fang, H., Zhou, H., Sanogo, S., Lipka, A. E., Fang, D. D., Percy, R. G., et al. (2014). Quantitative trait locus analysis of Verticillium wilt resistance in an
(2016YFD01014018); National Science and Technology Major Project for Transgenic Breeding (2014ZX0800501B).

\section{SUPPLEMENTARY MATERIAL}

The Supplementary Material for this article can be found online at: https://www.frontiersin.org/articles/10.3389/fpls.2017. 02272/full\#supplementary-material

Figure S1 Alignment of the Gbvdr6 of Hai7124 and its homology in TM-1. The red letters indicate the variant amino acids between the two genes.

introgressed recombinant inbred population of Upland cotton. Mol. Breed. 33, 709-720. doi: 10.1007/s11032-013-9987-9

Fradin, E. F., Adb-El-Haliem, A., Masini, L., van den Berg, G., Joosten, M., and Thomma, B. (2011). Interfamily transfer of tomato Vel mediates Verticillium resistance in Arabidopsis. Plant Physiol. 156, 2255-2265. doi: 10.1104/pp.111.180067

Fradin, E. F., Zhang, Z., Juarez Ayala, J. C., Castroverde, C. D., Nazar, R. N., Robb, J., et al. (2009). Genetic dissection of Verticillium wilt resistance mediated by tomato Ve1. Plant Physiol. 150, 320-332. doi: 10.1104/pp.109.136762

Fradin, E., Zhang, Z., Rovenich, H., Song, Y., Liebrand, T., and Arnold, D. (2014). Functional analysis of the tomato immune receptor Ve1 through domain swaps with its non-functional homolog Ve2. PLoS ONE 9:e88208. doi: 10.1371/journal.pone.0088208

Gao, X., Li, F., Li, M., Kianinejad, A. S., Dever, J. K., Wheeler, T. A., et al. (2013). Cotton GhBAK1 mediates Verticillium wilt resistance and cell death. J. Integr. Plant Biol. 55, 586-596. doi: 10.1111/jipb.12064

Gayoso, C., Pomar, F., Novo-Uzal, E., Merino, F., and llarduya OMD (2010). The $\mathrm{Ve}$-mediated resistance response of the tomato to Verticillium dahliae involves $\mathrm{H}_{2} \mathrm{O}_{2}$, peroxidase and lignins and drives PAL gene expression. BMC Plant Biol. 10:232. doi: 10.1186/1471-2229-10-232

Gibson, S. W., and Todd, C. D. (2015). Arabidopsis AIR12 influences root development. Physiol. Mol. Biol. Plants 21, 479-489. doi: 10.1007/s12298-015-0323-1

Hiratsu, K., Matsui, K., Koyama, T., and Ohme-Takagi, M. (2003). Dominant repression of target genes by chimeric repressors that include the EAR motif, a repression domain, in Arabidopsis. Plant J. 34, 733-739. doi: 10.1046/j.1365-313X.2003.01759.x

Humphries, J. A., Vejlupkova, Z., Luo, A. D., Meeley, R. B., Sylvester, A. W., Fowler, J. E., et al. (2011). ROP GTPases act with the receptor-like protein PAN1 to polarize asymmetric cell division in maize. Plant Cell 23, 2273-2284. doi: 10.1105/tpc.111.085597

Jefferson, R. A., Kavanagh, T. A. and Bevan, M. W. (1987). GUS fusions: $\mathrm{B}$-glucuronidase as a sensitive and versatile gene fusion marker in higher plants. EMBO J. 6, 3901-3907.

Jeong, S., Trotochaud, A. E., and Clark, S. E. (1999). The Arabidopsis CLAVATA2 gene encodes a receptor-like protein required for the stability of the CLAVATA1 receptor-like kinase. Plant Cell 11, 1925-1933. doi: 10.1105/tpc.11.10.1925

Jonge, R. D., Esse, H. P. V., Maruthachalam, K., Bolton, M. D., Santhanam, P., Saber, M. K., et al. (2012). Tomato immune receptor $V e 1$ recognizes effector of multiple fungal wilt pathogens uncovered by genome and RNA sequencing. Proc. Natl. Acad. Sci. U.S.A. 109, 5110-5115. doi: 10.1073/pnas.1119623109

Jun, Z., Zhang, Z., Zhou, L., Fang, L., Chen, X., et al. (2015). Overexpression of $G b R L K$, a putative receptor-like kinase gene, improved cotton tolerance to Verticillium wilt. Sci. Rep. 5:15048. doi: 10.1038/srep15048

Kawchuk, L. M., Hachey, J., Lynch, D. R., Kulcsar, F., van Rooijen, G., Waterer, D. R., et al. (2001). Tomato Ve disease resistance genes encode cell surface-like receptors. Proc. Natl. Acad. Sci. U.S.A. 98, 6511-6515. doi: 10.1073/pnas.091114198

Klosterman, S. J., Atallah, Z. K., Vallad, G. E., and Subbarao, K. V. (2009). Diversity, pathogenicity, and management of verticillium species. Annu. Rev. Phytopathol. 47, 39-62. doi: 10.1146/annurev-phyto-080508-081748

Klosterman, S. J., Subbarao, K. V., Kang, S. C., Veronese, P., Gold, S. E., Thomma, B. P., et al. (2011). Comparative genomics yields insights into 
niche adaptation of plant vascular wilt pathogens. PLoS Pathog. 7:e1002137. doi: 10.1371/journal.ppat.1002137

Kruijt, M., De Kock, M. J., and de Wit, P. J. (2005). Receptor-like proteins involved in plant disease resistance. Mol. Plant Pathol. 6, 85-97. doi: 10.1111/j.1364-3703.2004.00264.x

Laurie-Berry, N., Joardar, V., Street, I. H., and Kunkel, B. N. (2006). The Arabidopsis thaliana JASMONATE INSENSITIVE 1 gene is required for suppression of salicylic acid-dependent defenses during infection by Pseudomonas syringae. Mol. Plant Microbe Interact. 19, 789-800. doi: 10.1094/MPMI-19-0789

Li, C., He, X., Luo, X., Xu, L., Liu, L., Min, L., et al. (2014). Cotton WRKY1 mediates the plant defense-to-development transition during infection of cotton by Verticillium dahliae by activating jasmonate zim-domain1 expression. Plant Physiol. 166, 2179-2194. doi: 10.1104/pp.114.246694

Li, F., Fan, G., Lu, C., Xiao, G., Zou, C., Kohel, R. J., et al. (2015). Genome sequence of cultivated Upland cotton (Gossypium hirsutum TM-1) provides insights into genome evolution. Nat. Biotechnol. 33, 524-530. doi: 10.1038/nbt.3208

Liebrand, T. W., Kombrink, A., Zhang, Z., Sklenar, J., Jones, A. M., Robatzek, S., et al. (2014). Chaperones of the endoplasmic reticulum are required for Ve1-mediated resistance to Verticillium. Mol. Plant Pathol. 15, 109-117. doi: $10.1111 / \mathrm{mpp} .12071$

Liu, L. J., Sonbol, F. M., Huot, B., Gu, Y. N., Withers, J., Mwimba, M., et al. (2016). Salicylic acid receptors activate jasmonic acid signalling through a noncanonical pathway to promote effector-triggered immunity. Nat. Commun. 7:13099. doi: 10.1038/ncomms13099

Liu, T., Song, T., Zhang, X., Yuan, H., Su, L., Li, W., et al. (2014). Unconventionally secreted effectors of two filamentous pathogens target plant salicylate biosynthesis. Nat. Commun. 5:4686. doi: 10.1038/ncomms5686

Livak, K. J., and Schmittgen, T. D. (2001). Analysis of relative gene expression data using real-time quantitative PCR and the $2^{-\Delta \Delta C T}$. Methods $25,402-408$. doi: 10.1006/meth.2001.1262

Lorenzo, O., Chico, J. M., Sanchez-Serrano, J. J., and Solano, R. (2004). Jasmonateinsensitivel encodes a MYC transcription factor essential to discriminate between different jasmonate-regulated defense responses in Arabidopsis. Plant Cell 16, 1938-1950. doi: 10.1105/tpc.022319

Luo, X., Xie, C., Dong, J., Yang, X., and Sui, A. (2014). Interactions between Verticillium dahliae and its host: vegetative growth, pathogenicity, plant immunity. Appl. Microbiol. Biotechnol. 98, 6921-6932. doi: 10.1007/s00253-014-5863-8

Mazarei, M., Elling, A. A., Maier, T. R., Puthoff, D. P., and Baum, T. J. (2007). GmEREBP1 is a transcription factor activating defense genes in soybean and Arabidopsis. Mol. Plant Microbe Interact. 20, 107-119. doi: 10.1094/MPMI-20-2-0107

Miao, W., Wang, X., Li, M., Song, C., Wang, Y., Hu, D., et al. (2010). Genetic transformation of cotton with a harpin-encoding gene hpaXoo confers an enhanced defense response against different pathogens through a priming mechanism. BMC Plant Biol. 10:67. doi: 10.1186/1471-2229-10-67

Mo, H., Wang, X., Zhang, Y., Zhang, G., Zhang, J., and Ma, Z. (2015). Cotton polyamine oxidase is required for spermine and camalexin signalling in the defence response to Verticillium dahliae. Plant J. 83, 962-975. doi: $10.1111 /$ tpj.12941

Mur, L. A., Kenton, P., Atzorn, R., Miersch, O., and Wasternack, C. (2006). The outcomes of concentration-specific interactions between salicylate and jasmonate signaling include synergy, antagonism, and oxidative stress leading to cell death. Plant Physiol. 140, 249-262. doi: 10.1104/pp.105.072348

Nelson, B. K., Cai, X., and Nebenfuhr, A. (2007). A multicolored set of in vivo organelle markers for co-localization studies in Arabidopsis and other plants. Plant J. 51, 1126-1136. doi: 10.1111/j.1365-313X.2007.03212.x

Ning, Z., Zhao, R., Chen, H., Ai, N., Zhang, X., Zhao, J., et al. (2013). Molecular tagging of a major quantitative trait locus for broad-spectrum resistance to Verticillium wilt in upland cotton cultivar Prema. Crop Sci. 53, 2304-2312. doi: 10.2135/cropsci2012.12.0694

Pan, X., Li, Y., Zhang, H., Huang, R., Liu, W., Ming, J., et al. (2014). Expression of signalling and defence-related genes mediated by over-expression of JERF1, and increased resistance to sheath blight in rice. Plant Pathol. 63, 109-116. doi: 10.1111/ppa.12064

Parkhi, V., Kumar, V., Campbell, L. A. M., Bell, A. A., and Rathore, K. S. (2010). Expression of Arabidopsis NPR1 in transgenic cotton confers resistance to non-defoliating isolates of Verticillium dahliae but not the defoliating isolates. J. Phytopathol. 158, 822-825. doi: 10.1111/j.1439-0434.2010. 01714.x

Postma, J., Liebrand, T. W., Bi, G. Z., Evrard, A., Bye, R. R., Mbengue, M., et al. (2016). Avr4 promotes $C f-4$ receptor-like protein association with the BAK1/SERK3 receptor-like kinase to initiate receptor endocytosis and plant immunity. New Phytol. 210, 627-642. doi: 10.1111/nph.13802

Rajasekaran, K., Cary, J. W., Jaynes, J. M., and Cleveland, T. E. (2005). Disease resistance conferred by the expression of a gene encoding a synthetic peptide in transgenic cotton (Gossypium hirsutum L.) plants. Plant Biotechnol. J. 3, 545-554. doi: 10.1111/j.1467-7652.2005.00145.x

Shi, Y., Zhang, B., Liu A., Li, W., Li, J., Lu, Q., et al. (2016). Quantitative trait loci analysis of Verticillium wilt resistance in interspecific backcross populations of Gossypium hirsutum x Gossypium barbadense. BMC Genomics 17:877. doi: 10.1186/s12864-016-3128-x

Tamura, K., Stecher, G., Peterson, D., Filipski, A., and Kumar, S. (2013). MEGA6: molecular evolutionary genetics analysis version 6.0. Mol. Biol. Evol. 30, 2725-2729. doi: 10.1093/molbev/mst197

Thaler, J. S., Humphrey, P. T., and Whiteman, N. K. (2012). Evolution of jasmonate and salicylate signal crosstalk. Trends Plant Sci. 17, 260-270. doi: 10.1016/j.tplants.2012.02.010

Tian, J., Zhang, X., Liang, B., Li, S., Wu, Z., Wang, Q., et al. (2010). Expression of baculovirus anti-apoptotic genes p35 and op-iap in cotton (Gossypium hirsutum L.) enhances tolerance to verticillium wilt. PLoS ONE 5:e14218. doi: 10.1371/journal.pone.0014218

Tohidfar, M., Mohammadi, M., and Ghareyazie, B. (2005). Agrobacteriummediated transformation of cotton (Gossypium hirsutum) using a heterologous bean chitinase gene. Plant Cell Tiss. Org. Cult. 83, 83-96. doi: 10.1007/s11240-004-6155-2

Tomkins, J. P., Peterson, D. G., Yang, T. J., Main, D., Wilkins, T. A., Paterson, A. H., et al. (2001). Development of genomic resources for cotton (Gossypium hirsutum L.): BAC library construction, preliminary STC analysis, and identification of clones associated with fiber development. Mol. Breed. 8, 255-261. doi: 10.1023/A:1013798716098

Tong, X., Qi, J., Zhu, X., Mao, B., Zeng, L., Wang, B., et al. (2012). The rice hydroperoxide lyase OsHPL3 functions in defense responses by modulating the oxylipin pathway. Plant J. 71, 763-775. doi: 10.1111/j.1365-313X.2012.05027.x

Umbeck, P., Johnson, G., Barton, K., and Swain, W. (1987). Genetically transformed cotton (Gossypium-Hirsutum-L) plants. Bio-Technol. 5, 263-266. doi: 10.1038/nbt0387-263

Wang, P., Ning, Z., Lin, L., Chen, H., Mei, H., Zhao, J., et al. (2014). Genetic dissection of tetraploid cotton resistant to Verticillium wilt using interspecific chromosome segment introgression lines. Crop J. 2, 278-288. doi: 10.1016/j.cj.2014.06.007

Wang, Y., Chen, D., Wang, D., Huang, Q., Yao, Z., Liu, F., et al. (2004). Over-expression of Gastrodia anti-fungal protein enhances Verticillium wilt resistance in coloured cotton. Plant Breed. 123, 454-459. doi: 10.1111/j.1439-0523.2004.01005.x

Yang, C., Guo, W., Li, G., Gao, F., Lin, S., and Zhang, T. (2008). QTLs mapping for Verticillium wilt resistance at seedling and maturity stages in Gossypium barbadense L. Plant Sci. 174, 290-298. doi: 10.1016/j.plantsci.2007. 11.016

Yang, C. L., Liang, S., Wang, H. Y., Han, L. B., Wang, F. X., Cheng, H. Q., et al. (2015a). Cotton major latex protein 28 functions as a positive regulator of the ethylene responsive factor 6 in defense against Verticillium dahliae. Mol. Plant 8, 399-411. doi: 10.1016/j.molp.2014.11.023

Yang, Y., Zhang, Y., Ding, P., Johnson, K., Li, X., and Zhang, Y. (2012). The ankyrin-repeat transmembrane protein BDAl functions downstream of the receptor-like protein SNC2 to regulate plant immunity. Plant Physiol. 159, 1857-1865. doi: 10.1104/pp.112.197152

Yang, Y., Ling, X., Chen, T., Cai, L., Liu, T., Wang, J., et al. (2015b). A cotton Gbvdr5 gene encoding a leucine-rich-repeat receptor-like protein confers resistance to verticillium dahliae in transgenic arabidopsis and upland cotton. Plant Mol. Biol. Rep. 33, 987-1001. doi: 10.1007/s11105-014-0810-5

Yi, S. Y., Shirasu, K., Moon, J. S., Lee, S. G., and Kwon, S. Y. (2014). The activated SA and JA signaling pathways have an influence on flg22triggered oxidative burst and callose deposition. PLOS ONE 9:e88951. doi: 10.1371/journal.pone.0088951 
Zhang, J., Fang, H., Zhou, H., Sanogo, S., and Ma, Z. (2014). Genetics, breeding, and marker-assisted selection for Verticillium wilt resistance in cotton. Crop Sci. 54:1289. doi: 10.2135/cropsci2013.08.0550

Zhang, J., Sanogo, S., Flynn, R., Baral, J. B., Bajaj, S., Hughs, S., et al. (2012). Germplasm evaluation and transfer of Verticillium wilt resistance from Pima (Gossypium barbadense) to Upland cotton (G. hirsutum). Euphytica 187, 147-160. doi: 10.1007/s10681-011-0549-0

Zhang, L., Ni, H., Du, X., Wang, S., Ma, X. W., Nurnberger, T., et al. (2017). The Verticillium-specific protein VdSCP7 localizes to the plant nucleus and modulates immunity to fungal infections. New Phytol. 215, 368-381. doi: $10.1111 / \mathrm{nph} .14537$

Zhang, T., Hu, Y., Jiang, W., Fang, L., Guan, X., Chen, J., et al. (2015). Sequencing of allotetraploid cotton (Gossypium hirsutum L. acc. TM-1) provides a resource for fiber improvement. Nat. Biotechnol. 33, 531-537. doi: 10.1038/nbt.3207

Zhang, X., Wang, L., Xu, X., Cai, C., and Guo, W. (2014). Genome-wide identification of mitogen-activated protein kinase gene family in Gossypium raimondii and the function of their corresponding orthologs in tetraploid cultivated cotton. BMC Plant Biol. 14:345. doi: 10.1186/s12870-014-0345-9

Zhang, B., Yang, Y., Chen, T., Yu, W., Liu, T., Li, H., et al. (2012). Island cotton Gbve1 gene encoding a receptor-like protein confers resistance to both defoliating and non-defoliating isolates of Verticillium dahliae. PLoS ONE 7:e51091. doi: 10.1371/journal.pone.0051091

Zhang, Y., Wang, X. F., Ding, Z. G., Ma, Q., Zhang, G. R., Zhang, S. L., et al. (2013). Transcriptome profiling of Gossypium barbadense inoculated with Verticillium dahliae provides a resource for cotton improvement. BMC Genomics 14:637. doi: 10.1186/1471-2164-14-637

Zhang, Y., Wang, X., Yang, S., Chi, J., Zhang, G., and Ma, Z. (2011). Cloning and characterization of a Verticillium wilt resistance gene from Gossypium barbadense and functional analysis in Arabidopsis thaliana. Plant Cell Rep. 30, 2085-2096. doi: 10.1007/s00299-011-1115-x
Zhang, Z., Fradin, E., de Jonge, R., van Esse, H. P., Smit, P., Liu, C. M., et al. (2013a). Optimized agroinfiltration and virus-induced gene silencing to study Ve1-mediated Verticillium resistance in tobacco. Mol. Plant Microbe Interact. 26, 182-190. doi: 10.1094/MPMI-06-12-0161-R

Zhang, Z., Song, Y., Liu, C. M., and Thomma, B. P. (2014). Mutational analysis of the vel immune receptor that mediates verticillium resistance in tomato. PLoS ONE 9:e99511. doi: 10.1371/journal.pone.0099511

Zhang, Z., van Esse, H. P., van Damme, M., Fradin, E. F., Liu, C. M., and Thomma, B. P. (2013b). Ve1-mediated resistance against Verticillium does not involve a hypersensitive response in Arabidopsis. Mol. Plant Pathol. 14, 719-727. doi: 10.1111/mpp.12042

Zhou, H., Fang, H., Sanogo, S., Hughs, S. E., Jones, D. C., and Zhang, J. (2013). Evaluation of Verticillium wilt resistance in commercial cultivars and advanced breeding lines of cotton. Euphytica 196, 437-448. doi: 10.1007/s10681-013-1045-5

Zhu, X., Soliman, A., Islam, M. R., Adam, L. R., and Daayf, F. (2017). Verticillium dahliae's isochorismatase hydrolase is a virulence factor that contributes to interference with potato's salicylate and jasmonate defense signaling. Front. Plant Sci. 8:399. doi: 10.3389/fpls.2017.00399

Conflict of Interest Statement: The authors declare that the research was conducted in the absence of any commercial or financial relationships that could be construed as a potential conflict of interest.

Copyright (C) 2018 Yang, Chen, Ling and Ma. This is an open-access article distributed under the terms of the Creative Commons Attribution License (CC BY). The use, distribution or reproduction in other forums is permitted, provided the original author(s) or licensor are credited and that the original publication in this journal is cited, in accordance with accepted academic practice. No use, distribution or reproduction is permitted which does not comply with these terms. 